argininosuccinate $1014 \mu \mathrm{mol} / \mathrm{L}$, consistent with a diagnosis of argininosuccinic aciduria, subsequently confirmed on molecular genetic testing. She was discharged on oral sodium phenylbutyrate, sodium benzoate and L-arginine, with an appropriate dietary plan. To date, there is no evidence of neurological sequelae, likely attributable to prompt diagnosis and treatment.

Conclusion Argininosuccinic aciduria is a rare, autosomal recessive disorder of urea cycle metabolism caused by deficiency of argininosuccinic lyase. Clinical and biochemical manifestations depend on the severity of enzyme deficiency. In the neonatal variant, it typically presents with hyperammonaemia, encephalopathy and respiratory alkalosis. This case highlights the importance of considering inborn errors of metabolism in the assessment of a sick neonate.

\section{P432 ENCEPHALOPATHY IN TEENAGERS - A CHALLENGING ETIOLOGIC DIAGNOSIS}

${ }^{1}$ Tiago Magalhaes*, ${ }^{2}$ Teresa Campos, ${ }^{2}$ Esmeralda Rodrigues, ${ }^{2}$ Carla Vasconcelos, ${ }^{1}$ Manuel Fontoura, ${ }^{3}$ Laura Vilarinho, ${ }^{2}$ Elisa Leão Teles. ${ }^{1}$ Centro Materno Pediátrico - Centro Hospitalar São João, Porto, Portugal; ${ }^{2}$ Reference Center for Inherited Metabolic Disorders Centro Hospitalar São João, Porto, Portugal; ${ }^{3}$ National Health Institute - Dr. Ricardo Jorge, Porto, Portugal

\subsection{6/archdischild-2019-epa.768}

Introduction Inherited metabolic disorders due to intoxication provoked by highly toxic endogenous compounds are among the causes of encephalopathy. That is the case of urea cycle disorders in which ammonia's great neurotoxicity can frequently be lethal. Ornithine Transcarbamylase (OTC) Deficiency is an X-link genetic disorder and the most common disorder of the urea cycle. Presentation in heterozygote females is variable and can go from being asymptomatic to having mild chronic symptoms with severe exacerbations in any age.

Case report Fifteen year old female with sporadic headache since the age twelve without other relevant medical history. Due to persistent frontoparietal headache for two weeks, she was given topiramate. Despite medication, the headache worsened and dizziness, blurred vision, dysarthria, aphasia, asterixis, motor incoordination and lethargy developed. Trauma, recent infections or toxic ingestions were denied. Diagnostic workup in a private hospital with laboratory studies including urine toxic screening, complete blood count, hepatic and renal function and cerebral MRI were normal. On account of a suspected iatrogenic cause for encephalopathy, topiramate was suspended after two days and the patient was referred to a Pediatric Emergency Department. On admission CSF study was normal but blood tests showed hyperammonemia (229 $\mu \mathrm{mol} / \mathrm{L})$ with latter increase to $(359 \mu \mathrm{mol} / \mathrm{L})$ despite treating measures (endovenous fluids and discontinuation of protein intake); She was given sodium benzoate, sodium phenylbutyrate and arginine resulting in blood levels of ammonia returning to normal. The demonstration of elevated urinary orotic acid led to the probable diagnosis of OTC deficiency which was later confirmed by molecular diagnosis.
Discussion Serum ammonia levels sould be measured in every patient with unexplained altered mental status. Primary (urea clycle disorders) and secondary hyperammonemia alike are considered emergency situations therefore, treatment should never be delayed by diagnostic tests. On the other hand, both treatment and exacerbation preventions have an impact in prognosis regardless of age.

\section{P433 AN INTERESTING CASE DIAGNOSED AS BOTH PHENYLKETONURIA AND MATERNAL PHENYLKETONURIA}

Sibel Öz* ${ }^{*}$ Deniz Kor, Sebile Kılavuz, Fatma Derya Bulut, Berna Şeker Yılmaz , Fadli Demir , Tuğçe Özakçaoğlu Kartal, Gözde Atasever Yıldırım, Halise Neslihan Önenli Mungan. Cukurova University, Adana, Turkey

\subsection{6/archdischild-2019-epa.769}

Introduction Phenylketonuria (PKU) is the most common and autosomal recessively inherited metabolic disease due to the deficiency of phenylalanine hydroxylase (PAH). Elevated levels of phenylalanine are not only toxic for the children but also teratogenic for the fetus. Clinical findings of maternal phenylketonuria (MPKU) are intrauterine growth retardation, microcephaly, significant developmental delay, congenital cardiac anomalies, and some other structural defects. Poorly diet control and high levels of blood phenylalanine causes to this severe but preventable clinical syndrome.

Case report A seven-day-old girl admitted to the pediatric metabolism clinic with a suspicion of PKU after newborn screening program. She was born at 40 weeks of gestation with a 2300 gr birth weight and hospitalized for 2 days. Her parents were first degree cousins and the family history was unremarkable for an inherited metabolic disease, however the mother had two miscarriages. Physical examination revealed microcephaly, mild facial dysmorphism, and cardiac murmur in addition to intrauterine growth retardation. Her blood phenylalanine level was $1140 \mu \mathrm{mol} / \mathrm{dL}$ and she was diagnosed as moderate phenylketonuria. As a ventricular septal defect was detected with echocardiography and the mother was born before the national newborn screening program was available, the mother's blood Phe concentration was measured. Surprisingly her blood Phe level was $1614 \mu \mathrm{mol} / \mathrm{dL}$. The mother was a 25 years old woman be able to graduate from primary school and. complained about only concentration problems and forgetfulness. She had also blond hair and blue eyes on physical examination.

Conclusion Maternal phenylketonuria is a preventable public health problem which causes undesirable results like mentalmotor retardation and cardiac defects. Although maternal phenylketonuria is not completely coped with, after newborn screening program the incidence is decreased. A strict Phe restricted diet beginning before the pregnancy together with frequent controls of blood Phe levels are essential for management. Here, we report this case, as she was diagnosed both phenylketonuria and maternal phenylketonuria and in order to emphasize thinking MPKU in the presence of microcephaly, developmental delay, and cardiac defects. 